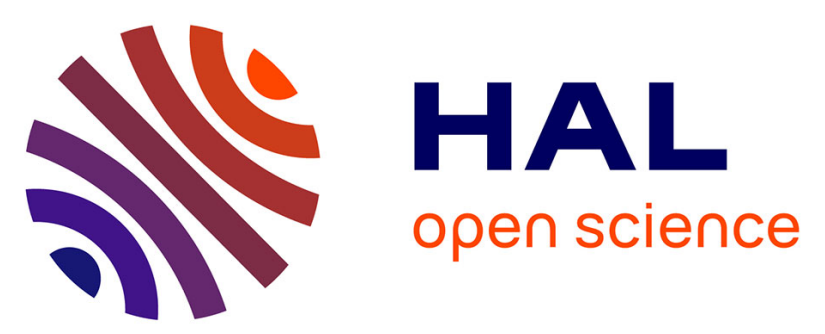

\title{
Generalized equation for transient-wave propagation in continuous inhomogeneous rigid-frame porous materials at low frequencies
}

Mohamed Fellah, Zine El Abiddine Fellah, Erick Ogam, F. G. Mitri, Claude Dépollier

\section{To cite this version:}

Mohamed Fellah, Zine El Abiddine Fellah, Erick Ogam, F. G. Mitri, Claude Dépollier. Generalized equation for transient-wave propagation in continuous inhomogeneous rigid-frame porous materials at low frequencies. Journal of the Acoustical Society of America, 2013, 134 (6), pp.4642. 10.1121/1.4824838 . hal-00881886

\section{HAL Id: hal-00881886 https://hal.science/hal-00881886}

Submitted on 14 May 2014

HAL is a multi-disciplinary open access archive for the deposit and dissemination of scientific research documents, whether they are published or not. The documents may come from teaching and research institutions in France or abroad, or from public or private research centers.
L'archive ouverte pluridisciplinaire HAL, est destinée au dépôt et à la diffusion de documents scientifiques de niveau recherche, publiés ou non, émanant des établissements d'enseignement et de recherche français ou étrangers, des laboratoires publics ou privés. 


\title{
Generalized equation for transient-wave propagation in continuous inhomogeneous rigid-frame porous materials at low frequencies.
}

\author{
M. Fellah
}

Laboratoire de Physique Théorique, Faculté de Physique, USTHB, BP 32 El Alia, Bab Ezzouar 16111, Algérie.

Z.E.A Fellah, E. Ogam

LMA, CNRS, UPR 7051, Aix-Marseille Univ, Centrale Marseille, F-13402 Marseille Cedex 20, France.

F.G. Mitri

Los Alamos National Laboratory, MPA-11, Sensors Electrochemical Devices, Acoustics Sensors Technology Team, MS D429, Los Alamos, NM 87545, USA.

C. Depollier

LUNAM Universite du Maine. UMR CNRS 6613 Laboratoire d'Acoustique de l'Universite du Maine UFR STS Avenue O. Messiaen 72085 Le Mans CEDEX 09 France.

Submitted to publication in the Journal of Acoustical Society of America. ${ }^{1}$

running title : inhomogeneous porous material

1. Special issue on Acoustics of Porous Media 


\section{ABSTRACT}

This paper provides a temporal model for the propagation of transient acoustic waves in continuous inhomogeneous isotropic porous material having a rigid frame at low frequency range. A temporal equivalent fluid model in which the acoustic wave propagates only in the fluid saturating the material, is considered. In this model, the inertial effects are described by the inhomogeneous inertial factor [A.N. Norris., J. Wave Mat. Interact. 1365 (1986)]. The viscous and thermal losses of the medium are described by two inhomogeneous susceptibility kernels which depend on the viscous and thermal permeabilities. The medium is one dimensional and its physical parameters (porosity, inertial factor, viscous and thermal permeabilities) are depth dependent. A generalized wave propagation equation in continuous inhomogeneous material is established and discussed. 


\section{INTRODUCTION}

The propagation of sound in fluid-saturated porous media with rigid solid frames is of great interest for a wide range of industrial applications. With air as the pore fluid ${ }^{1}$ applications can be found in noise control, nondestructive material characterization, thermoacoustically controlled heat transfer, etc.

The acoustic propagation in homogeneous porous materials having rigid frame has been well studied, different methods and techniques were developed in frequency ${ }^{1-5}$ and time domains ${ }^{6-12}$ for the acoustic characterization. All these techniques are valid only for homogeneous porous materials, in which, their physical parameters are constant inside the porous medium. However, in the general case, the porous media are inhomogeneous ${ }^{13-15}$ and their physical properties are locally constants, i.e. they are constant in the elementary volume of homogenization ${ }^{13}$, but they may vary from point to point in the porous medium. For this general case, a good understanding of the acoustic propagation is necessary for developing a new methods of characterization. A generalized hyperbolic fractional equation for transient wave propagation in inhomogeneous rigid-frame porous materials has been established in the asymptotic domain (high frequency range $)^{15}$, but not in the viscous domain (low frequency range), in which another set of physical parameters (inertial factor, viscous and thermal permeabilities) intervene in the propagation. The static thermal permeability ${ }^{16} k_{0}^{\prime}$ of the porous material is a geometrical parameter equal to the inverse trapping constant of the solid frame ${ }^{17}$. In the description of the thermal exchanges between the frame and the saturating fluid, the static thermal permeability plays a role similar to the viscous permeability in the description of the viscous forces. The inertial factor ${ }^{18} \alpha_{0}$ corresponds to the low frequency approximation of the dynamic tortuosity ${ }^{1,6}$ given by Norris ${ }^{18}$; $\alpha_{0}=\frac{\left\langle\mathbf{v}(\mathbf{r})^{2}\right\rangle}{\langle\mathbf{v}(\mathbf{r})\rangle^{2}}$, where $\langle\mathbf{v}(\mathbf{r})\rangle$ is the average velocity of the viscous fluid for direct current flow within a volume element, small compared to the relevant wavelength, but large compared to the individual grains/pores of the solid. 
This time-domain approach is an alternative to the classical frequency-domain model. It is an advantage of the time-domain $\operatorname{method}^{6-11,14,15,19-22}$ that the results are immediate and direct. The attraction of a time domain based approach is that analysis is naturally bounded by the finite duration of acoustic pressures and it is consequently the most appropriate approach for transient signals. However, for wave propagation generated by time harmonic incident waves and sources (monochromatic waves), the frequency analysis is more appropriate ${ }^{1-5}$.

This work follows the investigation previously done in Refs. 6 and 15, in which a time-domain approach was developed and a generalized hyperbolic fractional equation of propagation has been established in the asymptotic domain (high frequency range). Here, a general expression for the equation of wave propagation in continuous inhomogeneous porous medium is derived at viscous domain (low frequency range) .

The outline of this paper is as follows. Section II shows the equivalent fluid model, the relaxation functions describing the inertial, viscous and thermal interactions between fluid and structure are recalled. In this section, the connection between the temporal operators and wave propagation in rigid homogeneous porous media in the low frequency range is established. Finally, in Section III the analytical derivation of the general propagation equation is given in time domain. The different terms of this equation are discussed.

\section{THE EQUIVALENT FLUID MODEL}

In air saturated porous media, the structure is assumed to be motionless : the acoustic waves travel only in the fluid filling the pores. The wave propagation is described by the equivalent fluid model which is a particular case of the Biot's theory ${ }^{23}$. In this model, the interactions between the fluid and the structure are taken into account in two frequency dependent response factors which are the generalized susceptibilities : the dynamic tortuosity of the medium $\alpha(\omega)^{3}$ and the dynamic compressibility of the air included in the medium $\beta(\omega)^{1,16}$. These two response 
factors are complex functions which heavily depend on the frequency $f=\omega / 2 \pi, \omega$ is the angular frequency. These functions represent the deviation from the behavior of the fluid in the free space as the frequency increases. Their theoretical expressions are given by Johnson et $a l^{3}$, and Allard ${ }^{1}$ and Lafarge et $a l^{16}$ :

$$
\begin{aligned}
& \alpha(\omega)=\alpha_{\infty}\left(1+\frac{\phi \sigma}{i \omega \alpha_{\infty} \rho} \sqrt{1+i \frac{4 \alpha_{\infty}^{2} \eta \rho \omega}{\sigma^{2} \Lambda^{2} \phi^{2}}}\right) \\
& \beta(\omega)=\gamma-(\gamma-1)\left(1+\frac{\eta \phi}{i \omega \rho k_{0}^{\prime} P_{r}} \sqrt{1+i \frac{4 k_{0}^{\prime 2} \rho \omega P_{r}}{\eta \phi^{2} \Lambda^{\prime 2}}}\right)^{-1}
\end{aligned}
$$

where $i^{2}=-1, \gamma$ represents the adiabatic constant, $P_{r}$ the Prandtl number, $\alpha_{\infty}$ the tortuosity, $\sigma$ the flow resistivity, $\Lambda$ and $\Lambda^{\prime}$ the viscous and thermal characteristic lengths ${ }^{1,3}, \eta$ is the fluid viscosity, $\phi$ is the porosity and $\rho$ is the fluid density. This model was initially developed by Johnson $^{3}$, and completed by Allard ${ }^{1}$ by adding the description of thermal effects. Later on, Lafarge ${ }^{16}$ introduced the parameter $k_{0}^{\prime}$ which describes the additional damping of sound waves due to the thermal exchanges between fluid and structure at the surface of the pores. Generally the ration between $\Lambda^{\prime}$ and $\Lambda$ is between 1 and 3 .

The functions $\alpha(\omega)$ and $\beta(\omega)$ express the viscous and thermal exchanges between the air and the structure which are responsible of the sound damping in acoustic materials. These exchanges are due on the one hand to the fluid-structure relative motion and on the other hand to the air compressions-dilatations produced by the wave motion. The part of the fluid affected by these exchanges can be estimated by the ratio of a microscopic characteristic length of the media, as for example the sizes of the pores, to the viscous and thermal skin depth thickness $\delta=(2 \eta / \omega \rho)^{1 / 2}$ and $\delta^{\prime}=\left(2 \eta / \omega \rho P_{r}\right)^{1 / 2}$. For the viscous effects this domain corresponds to the region of the fluid in which the velocity distribution is perturbed by the frictional forces at the interface between the viscous fluid and the motionless structure. For the thermal effects, it is the fluid volume affected by the heat exchanges between the two phases of the porous medium, the solid skeleton being seen as a heat sink. At low frequencies (viscous domain) $)^{24}$, the viscous and thermal skin thicknesses are much larger than the radius of the pores $\delta / r \gg 1$ and $\delta^{\prime} / r \gg 1$. The viscous 
forces are important everywhere in the fluid, the compression dilatation cycle in the porous material is slow enough to favor the thermal interactions between fluid and structure. At the same time the temperature of the frame is practically unchanged by the passage of the sound wave because of the high value of its specific heat : the frame acts as a thermostat. In this case, the expressions of the dynamic tortuosity and compressibility are given by the relations ${ }^{24,25}$ :

$$
\begin{aligned}
& \alpha(\omega)=\alpha_{0}\left(1+\frac{\eta \phi}{j \omega \alpha_{0} \rho k_{0}}\right), \\
& \beta(\omega)=\gamma-\frac{(\gamma-1) \rho k_{0}^{\prime} P_{r}}{\eta \phi} j \omega,
\end{aligned}
$$

where $j^{2}=-1, \gamma$ represents the adiabatic constant, $P_{r}$ the Prandtl number, $k$ is the viscous permeability related to the flow resistivity $\sigma$ by the relation : $k=\eta / \sigma$.

In the time domain, the factors $\alpha(\omega)$ and $\beta(\omega)$ are operators and their asymptotic expressions are given by $^{24,25}$ :

$$
\begin{aligned}
& \tilde{\alpha}(t)=\alpha_{0}\left(\delta(t)+\frac{\eta \phi}{\alpha_{0} \rho k_{0}} \partial_{t}^{-1}\right), \\
& \tilde{\beta}(t)=\gamma \delta(t)-\frac{(\gamma-1) \rho k_{0}^{\prime} P_{r}}{\eta \phi} \partial_{t} .
\end{aligned}
$$

In these equations, $\partial_{t}^{-1}$ is the integral operator $\partial_{t}^{-1} g(t)=\int_{0}^{t} g\left(t^{\prime}\right) d t^{\prime}$. In each of these equations the first term in the right hand side is the instantaneous response of the medium $(\delta(t)$ is the Dirac function) while the second term is the memory function. In electromagnetism, the instantaneous response is called optical response. It describes all the processes which cannot be resolved by the signal.

In this framework, the basic equations of the acoustic waves propagation along the positive axis direction are :

$$
\begin{aligned}
& \rho \tilde{\alpha}(t) * \frac{\partial w}{\partial t}=-\phi \frac{\partial p}{\partial x} \\
& \frac{\phi \tilde{\beta}(t)}{K_{a}} * \frac{\partial p}{\partial t}=-\frac{\partial w}{\partial x} .
\end{aligned}
$$

The first equation is the Euler equation, the second one is the constitutive equation. $K_{a}$ is the bulk modulus of air, $p$ is acoustic pressure and $\mathbf{w}=\phi \mathbf{v}$ where $\mathbf{v}$ is the particle velocity, $*$ denotes 
the shorthand notation for the time convolution

$$
(f * g)(t)=\int_{0}^{t} f\left(t-t^{\prime}\right) g\left(t^{\prime}\right) d t^{\prime}
$$

The wave equation is deduced from these equations ${ }^{24,25}$ :

$$
\frac{\partial^{2} p(x, t)}{\partial x^{2}}-\frac{1}{c^{2}} \frac{\partial^{2} p(x, t)}{\partial t^{2}}-A \frac{\partial p(x, t)}{\partial t}+B \frac{\partial^{3} p(x, t)}{\partial t^{3}}=0
$$

where the coefficients $c, A$ and $B$ are constants respectively given by;

$$
\frac{1}{c^{2}}=\frac{\rho}{K_{a}}\left(\alpha_{0} \gamma-\frac{(\gamma-1) P_{r} k_{0}^{\prime}}{k_{0}}\right), \quad A=\frac{\eta \phi \gamma}{K_{a} k_{0}}=\frac{\phi \sigma \gamma}{K_{a}}, \quad B=\frac{\rho^{2}(\gamma-1) k_{0}^{\prime} P_{r} \alpha_{0}}{K_{a} \eta \phi}
$$

the first one is related to the wavefront velocity $c=1 / \sqrt{\rho\left(\alpha_{0} \gamma-\frac{(\gamma-1) P_{r} k_{0}^{\prime}}{k_{0}}\right) / K_{a}}$ of the wave in the air included in the porous material. The term $\left(\alpha_{0} \gamma-\frac{(\gamma-1) P_{r} k_{0}^{\prime}}{k_{0}}\right)$ appears as the refractive index of the medium which changes the wave velocity from $c_{0}=\sqrt{K_{a} / \rho}$ in free space to $c=c_{0} / \sqrt{\left(\alpha_{0} \gamma-\frac{(\gamma-1) P_{r} k_{0}^{\prime}}{k_{0}}\right)}$ in the porous medium. The originality of this wavefront velocity is its dependence on the inertial, viscous and thermal effects in the porous material, compared to the wavefront velocity in high frequency range $6,10,11,15$ which depends only on inertial interactions via the tortuosity $\alpha_{\infty}$. The coefficient $A$ is responsible of the attenuation of the wave without dispersion due to the viscous losses via the viscous permeability $k_{0}$. The constant $B$ governs the spreading of the signal, and describes the dispersion due to the thermal interactions between fluid and structure via the thermal permeability $k_{0}^{\prime}$. To note that in this regime of frequency, the dispersion phenomena describing by the term $B \partial^{3} p(x, t) / \partial t^{3}$ is not as important as in the high frequency range, in which the fractional derivatives ${ }^{6,10,11,15}$ are needed to describe in time domain the high dispersion in the porous material. This propagation equation has been solved analytically in Ref. 24 . The direct ${ }^{24}$ and inverse ${ }^{25-27}$ scattering problem for a slab of porous material has been studied given a good estimation of the physical parameters (viscous and thermal permeabilities, and inertial factor). 


\section{GENERALIZED PROPAGATION EQUATION IN INHO- MOGENEOUS POROUS MATERIALS}

Consider the propagation of transient acoustic waves in continuous inhomogeneous porous material having rigid frame. In this material, the acoustical parameters (inertial factor, porosity, viscous and thermal permeability) depend on the thickness, and are continuous functions. For a wave propagating along the $x$-axis, the fluid-structure interactions are described by the inhomogeneous relaxation operators $\alpha(x, t)$ and $\beta(x, t)$ given by

$$
\begin{aligned}
& \tilde{\alpha}(x, t)=\alpha_{0}(x)\left(\delta(t)+\frac{\eta \phi(x)}{\alpha_{0}(x) \rho k(x)} \partial_{t}^{-1}\right), \\
& \tilde{\beta}(x, t)=\gamma \delta(t)-\frac{(\gamma-1) \rho k^{\prime}(x) P_{r}}{\eta \phi(x)} \partial_{t} .
\end{aligned}
$$

In these equations, the porosity $\phi(x)$, the tortuosity $\alpha_{0}(x)$, viscous and thermal permeability $k(x)$ and $k^{\prime}(x)$ depend on the thickness of the porous material for describing the inhomogeneous losses in the material.

In this framework, the basic equations ${ }^{13-15}$ for our model can be written as

$$
\begin{aligned}
& \rho \alpha(x, t) * \frac{\partial w(x, t)}{\partial t}=-\phi(x) \frac{\partial p(x, t)}{\partial x}, \\
& \frac{\phi(x)}{K_{a}} \beta(x, t) * \frac{\partial p(x, t)}{\partial t}=-\frac{\partial w(x, t)}{\partial x} .
\end{aligned}
$$

In the next section, the generalized propagation equation in continuous inhomogeneous porous material having an acoustical parameters varying with depth is derived. The derivation of the generalized wave equation in an inhomogeneous porous material is important for computing the propagation of an acoustic pulse inside the medium, and for solving the direct and inverse scattering problems.

Let us consider the Euler equation (14) and the constitutive one (15) in an infinite inhomogeneous porous material. By putting

$$
a(x)=\frac{\eta \phi(x)}{\alpha_{0}(x) \rho k(x)} \quad \text { and } \quad b(x)=\frac{(\gamma-1) \rho k^{\prime}(x) P_{r}}{\eta \phi(x)}
$$


we obtain :

$$
\begin{aligned}
& \rho \alpha_{0}(x) \delta(t) * \frac{\partial w(x, t)}{\partial t}+a(x) w(x, t)=-\phi(x) \frac{\partial p(x, t)}{\partial x} \\
& \frac{\phi(x)}{K_{a}} \gamma \delta(t) * \frac{\partial p(x, t)}{\partial t}-b(x) \frac{\partial^{2} p(x, t)}{\partial t^{2}}=-\frac{\partial w(x, t)}{\partial x} .
\end{aligned}
$$

We note $P(x, z)$ the Laplace transform of $p(x, t)$ defined by

$$
P(x, z)=\mathcal{L}[p(x, t)]=\int_{0}^{\infty} \exp (-z t) p(x, t) d t
$$

The Laplace transform of Eqs. 16, 17 yields

$$
\begin{aligned}
& \rho \alpha_{0}(x)\left[1+\frac{a(x)}{z}\right] z W(x, z)=-\phi(x) \frac{\partial P(x, z)}{\partial x}, \\
& \frac{\phi(x)}{K_{a}}[\gamma-b(x) z] z P(x, z)=-\frac{\partial W}{\partial x}(x, z)
\end{aligned}
$$

where $W(x, z)$ is the Laplace transform of $w(x, t)$.

Using Eqs. 19 and 20 and the calculus developed in Appendix. A, we obtain the following equation

$$
\begin{aligned}
& \frac{\partial^{2} P(x, z)}{\partial x^{2}}=\left[\frac{\partial}{\partial x} \ln \frac{\alpha_{0}(x)}{\phi(x)}+\frac{\partial a(x)}{\partial x}\left(\frac{1}{z+a(x)}\right)\right] \frac{\partial P(x, z)}{\partial x} \\
& +\frac{\rho \alpha_{0}(x)}{K_{a}}\left[-b(x) z^{3}+(\gamma-a(x) b(x)) z^{2}+\gamma a(x) z\right] P(x, z) .
\end{aligned}
$$

Using the inverse Laplace transform of Eq. 21 and the initial conditions ${ }^{10} ; \frac{\partial p}{\partial t}(x, 0)=p(x, 0)=0$, we find the generalized propagation equation in time domain.

$$
\begin{aligned}
& \frac{\partial^{2} p(x, t)}{\partial x^{2}}-\frac{1}{c^{2}(x)} \frac{\partial^{2} p(x, t)}{\partial t^{2}}-A^{\prime}(x) \frac{\partial p(x, t)}{\partial t}+B^{\prime}(x) \frac{\partial^{3} p(x, t)}{\partial t^{3}} \\
& -\frac{\partial a(x)}{\partial x} \int_{0}^{t} \exp (-\tau a(x)) \frac{\partial p(x, t-\tau)}{\partial x} d \tau-\frac{\partial}{\partial x}\left[\ln \frac{\alpha_{0}(x)}{\phi(x)}\right] \frac{\partial p(x, t)}{\partial x}
\end{aligned}
$$

where

$\frac{1}{c^{2}(x)}=\frac{\rho \alpha_{0}(x)[\gamma-a(x) b(x)]}{K_{a}}, \quad A^{\prime}(x)=\frac{\rho \alpha_{0}(x) \gamma a(x)}{K_{a}}, \quad$ and $\quad B^{\prime}(x)=\frac{\rho \alpha_{0}(x) b(x)}{K_{a}}$

Eq. (22) is the generalized propagation equation for lossy inhomogeneous porous material in low frequency range. This equation is very important for treating the direct and inverse scattering 
problems in inhomogeneous porous materials in time domain. It is easy to find the special case of homogeneous porous medium, i.e. when $\alpha_{0}(x), \phi(x), k(x)$ and $k^{\prime}(x)$ become constants (independent of $x$ ), we find $A^{\prime}(x)=A(x), B^{\prime}(x)=B, \partial a(x) / \partial x=0$. In this case, the generalized wave propagation (Eq. 22) is reduced to the propagation equation in homogeneous material (Eq. 10).

The first and second term in the propagation equation $(22): \frac{\partial^{2} p}{\partial x^{2}}(x, t)-\frac{1}{c^{2}(x)} \frac{\partial^{2} p}{\partial t^{2}}(x, t)$ describe the propagation (time translation) via the front wave velocity $c(x)$. The term $\sqrt{\left(\alpha_{0}(x) \gamma-\frac{(\gamma-1) P_{r} k^{\prime}(x)}{k(x)}\right)}$ appears as the refractive index of the medium which changes the wave velocity from $c_{0}=\sqrt{K_{a} / \rho}$ in free space to $c=c_{0} / \sqrt{\left(\alpha_{0}(x) \gamma-\frac{(\gamma-1) P_{r} k^{\prime}(x)}{k(x)}\right)}$ in the porous medium. From this equation, (as it has been shown in the homogeneous $\operatorname{case}^{24}$ ), it can be seen that the inertial, viscous and thermal effects are all responsible of the change in the wave front velocity compared to the high frequency inhomogeneous case ${ }^{15}$ in which only the inertial effect modify the front wave velocity. The third term in the propagation equation $(22): A^{\prime}(x) \frac{\partial p(x, t)}{\partial t}$ is the most important one for describing the acoustic attenuation in porous materials at low frequency range ${ }^{26}$, it results on the attenuation of the wave without dispersion. It depends on the viscous permeability, which is the most influential parameter in this domain of frequency. This term describes the acoustic attenuation due to the viscous and inertial interactions between fluid and structure. To note that the thermal effects do not intervene in this therm. This can be explained by the fact that the viscous effects are the most important at this range of frequency. The contribution of the thermal effects is taken into account only in the second term in Eq. 4. This term is very sensitive to the spatial variation of the viscous permeability $k(x)$.

The fourth term in the propagation equation $(22): B^{\prime}(x) \frac{\partial^{3} p(x, t)}{\partial t^{3}}$ governs the spreading of the signal, and describes the weak dispersion due to the thermal interactions between fluid and structure via the spatial thermal permeability $k^{\prime}(x)$. To note that in this regime of frequency, the dispersion phenomena described by this term are not as important as in the high frequency range, in which the fractional derivatives are needed to describe in the time domain the high 
dispersion in the porous material.

The final term : $\frac{\partial}{\partial x}\left[\ln \frac{\alpha_{0}(x)}{\phi(x)}\right] \frac{\partial p(x, t)}{\partial x}$ describes the attenuation caused by the spatial variation of the tortuosity and the porosity. In contrast to the other terms, this term does not contains temporal derivative of the pressure, it is independent of the relaxations times of the medium and thus to the frequency component of the acoustic signal.

Finally the term in the propagation equation (22) : $-\frac{\partial a(x)}{\partial x} \int_{0}^{t} \exp (-\tau a(x)) \frac{\partial p(x, t-\tau)}{\partial x} d \tau$ describes the spatial variation of the inhomogeneity of the porous medium due to the inertial and viscous interactions (there are no thermal effects) of the medium.

The generalized propagation equation derived at the viscous domain (low frequency range) and given by Eq. 22 is very different from the generalized fractional equation derived at the asymptotic domain (high frequency range) ${ }^{15}$. The physical parameters describing the propagation are not the same in the two domains, for example, the viscous and thermal interactions between fluid and structure are described by the viscous and thermal characteristic lengths $\Lambda$ and $\Lambda^{\prime}$ at the asymptotic domain, however at the viscous domain, these interactions are described by the viscous and thermal permeabilities $k_{0}$ and $k_{0}^{\prime}$. The inertial effects are also described by different parameters at the two regimes of frequencies, the tortuosity $\alpha_{\infty}$ is used for the high frequency range, while the inertial factor $\alpha_{0}$ is used at the low frequency range. In addition the relaxations times responsible of the dispersion phenomenon and memory effects of the acoustic wave are expressed by different temporal operators. It has been shown ${ }^{6,15}$ in the asymptotic domain (high frequency range), that the tortuosity and compressibility operators depend on fractional operators for describing the viscous and thermal interactions. These fractional operators give a fractional derivative term in the generalized propagation equation for the inhomogeneous material. This fractional term is not found in the derived equation (equation 22). For the viscous domain corresponding to the low frequency range, the loss operator have a simple expressions, which are functions of simple derivatives, and thus there is no fractional term in the generalized propagation equation. However, we find a term with third derivative responsible of the disper- 
sion which is not found in the asymptotic domain. To note that the dispersion phenomenon described by the fractional derivative ${ }^{10,11}$ is more important in the asymptotic domain than the dispersion phenomenon described by the third derivative term ${ }^{24,25}$ in the viscous domain. The porosity is the only parameter which plays an important role in both the high and low frequencies domains. The variation of the porosity with the depth $\phi(x)$ is found in the two generalize equations (asymptotic and viscous domains).

Generally it is interesting to work at the very low frequencies, especially when we want to obtain the viscous permeability or the flow resistivity by solving the inverse problem directly in time domain via transmitted or reflected acoustic waves ${ }^{25-27}$. In this case, the effect of the inertial factor $\alpha_{0}$ becomes negligible in the expression of the inhomogeneous relaxation operator $\tilde{\alpha}(x, t)$ given by Eq. 12, in this case, we obtain the following expression :

$$
\tilde{\alpha}(x, t)=\frac{\eta \phi(x)}{\rho k(x)} \partial_{t}^{-1}
$$

The viscous interactions are the most important in this case, the inertial exchange between fluid and structure are negligible. The thermal permeability is not involved in the basic equations of acoustic in porous material in this domain of frequency. The inhomogeneous relaxation operator $\tilde{\beta}(x, t)$ becomes independent of the depth $x$, its expression will be given by

$$
\tilde{\beta}(t)=\gamma \delta(t)
$$

In this framework, the basic equations of the model become

$$
\begin{aligned}
& \frac{\eta \phi(x)}{k(x)} w(x, t)=-\phi(x) \frac{\partial p(x, t)}{\partial x} \\
& \frac{\gamma \phi(x)}{K_{a}} \frac{\partial p(x, t)}{\partial t}=-\frac{\partial w(x, t)}{\partial x}
\end{aligned}
$$

where the Euler equation (23) is reduced to Darcy's law which defines the variation of the static flow resistivity with the depth $x ; \sigma(x)=\eta / k(x)$. The wave equation in time domain is given by

$$
\frac{\partial^{2} p(x, t)}{\partial x^{2}}+\left[\frac{\partial}{\partial x} \ln \left(\frac{k(x)}{\eta}\right)\right] \frac{\partial p(x, t)}{\partial x}-\frac{\gamma \eta \phi(x)}{K_{a} k(x)} \frac{\partial p(x, t)}{\partial t}=0
$$


The fields which are varying in time, the pressure, the acoustic velocity, etc., follow a diffusion equation with the diffusion constant

$$
D(x)=\frac{K_{a} k(x)}{\gamma \eta \phi(x)}
$$

A quite similar result is given in Refs. 6, 26 and 27 for the homogeneous porous materials. For liquid saturated porous materials, the thermal expansion is neglected, and thus the adiabatic constant does not appear in the Johnson' ${ }^{28}$ model for homogeneous porous materials. We recall that in the homogeneous case, we have the following diffusion equation :

$$
\frac{\partial^{2} p(t)}{\partial x^{2}}-\left(\frac{\gamma \eta \phi}{K_{a} k}\right) \frac{\partial p(t)}{\partial t}=0
$$

The main difference between Eqs. 25 and 26 is the depth dependence of the porosity $\phi(x)$ and the viscous permeability $k(x)$. In addition, the term $: \frac{\partial}{\partial x} \ln \left(\frac{k(x)}{\eta}\right) \frac{\partial p(x, t)}{\partial x}$ describes the attenuation caused by the spatial variation of the permeability $k(x)$ due to the inhomogeneity of the porous material. At the very low frequencies, the generalized propagation equation (22) is reduced to the diffusion equation (25). These equations are very important for the solving the direct and inverse problem in time domain for the inhomogeneous porous materials. This is our objective in the future works.

\section{CONCLUSION}

In this paper the generalized wave equation in inhomogeneous porous material is established in the viscous domain (low frequency range when $\delta / r \gg 1$ and $\delta^{\prime} / r \gg 1$ ). The different terms of the propagation equation show how the spatial variation of the inertial factor, porosity, viscous and thermal permeabilities affect the propagation. In this propagation equation there is no fractional derivatives as it has been shown in the asymptotic domain ${ }^{15}$ (high frequency domain), but a third derivative term is needed for describing the dispersion in the porous material. At low frequencies, the inertial, viscous and thermal effects are involved in the expression of the velocity 
of the wavefront. The domain of very low frequency range is also studied and a generalized diffusion equation is derived.

These established equations constitute a basis for the resolution of the direct and inverse scattering problems. Future studies will concentrate on methods and inversion algorithms to optimize the acoustic properties of inhomogeneous porous media. 


\section{APPENDIX. A}

By differentiating both sides of Eq. 19 with respect to $\mathrm{x}$, one finds that

$$
\begin{aligned}
& \rho \frac{\partial \alpha_{0}(x)}{\partial x}[z+a(x)] W(x, z)+\rho \alpha_{0}(x) \frac{\partial a(x)}{\partial x} W(x, z) \\
& +\rho \alpha_{0}(x)[z+a(x)] \frac{\partial W(x, z)}{\partial x}=-\phi(x) \frac{\partial^{2} P(x, z)}{\partial x^{2}}-\frac{\partial P(x, z)}{\partial x} \frac{\partial \phi(x)}{\partial x} .
\end{aligned}
$$

From Eq. 19, we obtain

$$
W(x, z)=\frac{-\phi(x)}{\rho \alpha_{0}(x)[z+a(x)]}\left(\frac{\partial P(x, z)}{\partial x}\right)
$$

The two first terms of Eq. 27 can be written as

$$
\begin{aligned}
& \rho \frac{\partial \alpha_{0}(x)}{\partial x}[z+a(x)] W(x, z)=-\frac{\partial \alpha_{0}(x)}{\partial x} \frac{\phi(x)}{\alpha_{0}(x)} \frac{\partial P(x, z)}{\partial x} \\
& \rho \alpha_{0}(x) \frac{\partial a(x)}{\partial x} W(x, z)=-\frac{\partial a(x)}{\partial x} \frac{\phi(x)}{z+a(x)} \frac{\partial P(x, z)}{\partial x}
\end{aligned}
$$

Using Eq. 20, the third term of Eq. 27 can be written as :

$$
\rho \alpha_{0}(x)[z+a(x)] \frac{\partial W(x, z)}{\partial x}=-\frac{\rho \alpha_{0}(x) \phi(x)}{K_{a}}[z+a(x)]\left[\gamma z-b(x) z^{2}\right] P(x, z)
$$

Eq. 27 becomes :

$$
\begin{aligned}
& {\left[-\frac{\partial \alpha_{0}(x)}{\partial x} \frac{\phi(x)}{\alpha_{0}(x)}-\frac{\partial a(x)}{\partial x}\left(\frac{\phi(x)}{z+a(x)}\right)\right] \frac{\partial P(x, z)}{\partial x}-\frac{\rho \alpha_{0}(x) \phi(x)}{K_{a}}[z+a(x)]\left[\gamma z-b(x) z^{2}\right] P(x, z)} \\
& =-\frac{\partial \phi(x)}{\partial x} \frac{\partial P(x, z)}{\partial x}-\phi(x) \frac{\partial^{2} P(x, z)}{\partial x^{2}}
\end{aligned}
$$

By dividing the two members of Eq.29 by $-\phi(x)$, we obtain the relation (21). 


\section{REFERENCES}

${ }^{1}$ J.F. Allard and N. Atalla, in Propagation of Sound in Porous Media : Modeling Sound Absorbing Materials (Wiley, Chichester, UK, 2009), Chap. 5.

${ }^{2}$ K. Attenborough, "On the acoustic slow wave in air-filled granular media," J. Acoust. Soc. Am. 81, 93-102 (1987).

${ }^{3}$ D.L. Johnson, J. Koplik and R. Dashen, "Theory of dynamic permeability and tortuosity in fluid-saturated porous media", J. Fluid.Mech. 176, 379-402 (1987).

${ }^{4}$ P. Leclaire, L. Kelders, W. Lauriks, N. R. Brown, M. Melon, and B. Castagnède, "Determination of viscous and thermal characteristics lengths of plastic foams by ultrasonic measurements in helium and air," J. Appl. Phys. 80, 2009-2012 (1996).

${ }^{5}$ C. Ayrault, A. Moussatov, B. Castagnède, and D. Lafarge, "Ultrasonic characterization of plastic foams via measurements with static pressure variations," Appl. Phys. Lett. 74, 32243226 (1999).

${ }^{6}$ Z.E.A. Fellah and C. Depollier, "Transient wave propagation in rigid porous media : a time domain approach", J. Acoust. Soc. Am., 107, 683-688 (2000).

${ }^{7}$ D.K. Wilson, V.E. Ostashev, S.L. Collier, "Time domain equations for sound propagation in rigid-frame porous media", J. Acoust. Soc. Am., 107, 1889-1892 (2004).

${ }^{8}$ Olga Umnova and Diego Turo, "Time domain formulation of the equivalent fluid model for 
rigid porous media (L)", J. Acoust. Soc. Am., 125, 1860-1863 (2009).

${ }^{9}$ Diego Turo, Olga Umnova, "Time Domain Modelling of Sound Propagation in Porous Media and the Role of Shape Factors", ACTA ACUSTICA UNITED WITH ACUSTICA, 96, 225-238 (2010).

${ }^{10}$ Z.E.A. Fellah, M. Fellah, W. Lauriks, C. Depollier, J.Y. Chapelon and Y.C. Angel, "Solution in time domain of ultrasonic propagation equation in porous material", Wave Motion, 38 $151-163(2003)$.

${ }^{11}$ Z.E.A Fellah, M. Fellah, W. Lauriks and C. Depollier 2003, "Direct and inverse scattering of transient acoustic waves by a slab of rigid porous material", J. Acoust. Soc. Am. 113 61-72 (2003).

${ }^{12}$ D. K. Wilson, "Simple, Relaxational Models for the Acoustical Properties of Porous Media", Appl. Acoust. 50 171-188 (1997).

${ }^{13}$ J.M. Carcione, Wave fields in real media : wave propagation in anisotropic, anelastic and porous media, (K. Helbig and S. Treitel, Pergamon, 2001), pp. 261-266.

${ }^{14}$ C. Depollier, Z.E.A. Fellah and M. Fellah 2004, "Propagation of transient acoustic waves in layered porous media : Fractional equations for scattering operators", J. Non Linear Dynamics. 38 181-190 (2004).

${ }^{15}$ M. Fellah, Z.E.A. Fellah and C. Depollier, "Generalized hyperbolic fractional equation for transient-wave propagation in layered rigid-frame porous materials", Phys. Rev. E. 77 016601-5 
(2008).

${ }^{16}$ D. Lafarge, P. Lemarinier, J. F. Allard, V. Tarnow, "Dynamic compressibility of air in porous structures at audible frequencies" J. Acoust. Soc. Am. 102, 1995-2006 (1997).

${ }^{17}$ M. Avellaneda and S. Torquato, "Rigourous link between fluid permeability, electrical conductivity and relaxation times for transport in porous media" Phys. Fluids A 3, 2529-2540 (1991).

${ }^{18}$ A.N. Norris, "On the viscodynamic operator in Biot's equations of poroelasticity", J. Wave Mat. Interact. 1 365-380 (1986).

${ }^{19}$ T.L. Szabo, "Time domain wave equations for lossy media obeying a frequency power law", J. Acoust. Soc. Am. 96, 491-500, (1994).

${ }^{20}$ G. Caviglia and A. Morro, "A closed-form solution for reflection and transmission of transient waves in multilayers", J. Acoust. Soc. Am. 116, 643-654 (2004).

${ }^{21}$ G. V. Norton and J. C. Novarini, "Including dispersion and attenuation directly in time domain for wave propagation in isotropic media", J. Acoust. Soc. Am. 113, 3024-3031 (2003).

${ }^{22}$ F. Prieur and S. Holm, "Nonlinear acoustic wave equations with fractional loss operators", J. Acoust. Soc. Am. 130, 1125-1132, (2011).

${ }^{23}$ M.A. Biot, "The theory of propagation of elastic waves in fluid-saturated porous solid. I. Low frequency range", J. Acoust. Soc. Am., 28, 168-178 (1956). 
${ }^{24}$ Z.E.A. Fellah, M. Fellah, F.G. Mitri, N. Sebaa, W. Lauriks and C. Depollier, "Transient acoustic wave propagation in air-saturated porous media at low frequencies", J. Appl. Phys. 102, 084906 (2007).

${ }^{25}$ M. Sadouki, M. Fellah, Z.E.A. Fellah, E.Ogam, N. Sebaa, F.G. Mitri and C. Depollier, "Measuring static thermal permeability and inertial factor of rigid porous materials", J. Acoust. Soc. Am., 130, 2627-2630 (2011).

${ }^{26}$ Z.E.A. Fellah, M. Fellah, N. Sebaa, W. Lauriks and C. Depollier, "Measuring flow resistivity of porous materials at low frequencies range via acoustic transmitted waves", J. Acoust. Soc. Am. 119, 1926-1928 (2006).

${ }^{27}$ N. Sebaa, Z.E.A. Fellah, M. Fellah, W. Lauriks and C. Depollier, "Measuring flow resistivity of porous material via acoustic reflected waves", J. Appl. Phys. 98, 084901 (2005).

${ }^{28}$ D. J. Johnson, "Recent developments in the acoustic properties of porous medium," in Proceedings of the International School of Physics Enrico Fermi, Course XCIII, edited by D. Sette (North-Holland, Amsterdam, 1986), pp. 255-290. 